Nat Rev Endocrinol. 2016 December 08; 13(1): 7-9. doi:10.1038/nrendo.2016.194.

\title{
The emotional cost of contraception
}

\author{
Rachel A. Ross and \\ Department of Psychiatry, Beth Israel Deaconess Medical Center, 330 Brookline Avenue, Boston, \\ Massachusetts 02215 and the Center for Anxiety and Traumatic Stress Disorders, Department of \\ Psychiatry, Massachusetts General Hospital, 1 Bowdoin Square, Boston, Massachusetts 02114, \\ USA
}

\author{
Ursula B. Kaiser \\ Division of Endocrinology, Diabetes and Hypertension, Brigham and Women's Hospital and \\ Harvard Medical School, 221 Longwood Avenue, Boston, Massachusetts 02115, USA
}

\section{Abstract}

The link between mood disorders and hormonal fluctuations has long been known, but the direction of this correlation has been questioned. New research suggests that initiation of hormonal contraception might lead to increased risk of first-time diagnosis of or treatment for depression over a short time frame, particularly for adolescents.

In the context of a research field populated by many conflicting reports, Skovlund et al. ${ }^{1}$ have produced the first prospective cohort study that attempts to identify a causal relationship between hormonal contraception and mood disorders in women. The investigators combined data from two Danish registries, the National Prescription Register and the Psychiatric Central Research Register, to create an expansive cohort. The rate ratios (that is, the relative risks) of first diagnosis of depression or treatment with an antidepressant were compared between women who had been prescribed hormonal contraception and women who were not using hormonal contraception. Given that rates of depression are higher among women than men $^{2}$ and that millions of women use some form of hormonal contraception (including, in some cases, for treatment of mood disorders), understanding the association between these two clinical entities is tremendously important.

The association between mood changes and hormones is not a new observation. Physicians are familiar with the increases in depressive symptoms during menopause, the emotional

\footnotetext{
Correspondence to U.B.K. ukaiser@bwh.harvard.edu.

Competing interests statement

The authors declare no competing interests.

Subject ontology terms

Health sciences/Diseases/Reproductive disorders/Endocrine reproductive disorders [URI/692/699/2732/2730]

Health sciences/Diseases/Psychiatric disorders/Depression [URI/692/699/476/1414]

Health sciences/Medical research/Outcomes research [URI/692/308/409]

Health sciences/Health care/Therapeutics/Hormonal therapies [URI/692/700/565/238]
} 
lability preceding menses for many women and the subtype of depression known as premenstrual dysphoric disorder. This latter diagnosis refers to a time-limited and hormonelinked depression for which brief intermittent antidepressant treatment or hormonal contraception is recommended ${ }^{3,4}$. Prior work through case reports, small cohort studies, systematic reviews and meta-analyses have espoused opposing ideas: some find contraceptives beneficial and some find them detrimental to mood ${ }^{5,6}$. These findings have been interpreted to suggest a role for exogenous progestins specifically (corresponding to the elevations in endogenous progesterone in the luteal phase of the menstrual cycle, linked to premenstrual dysphoric disorder) in causing depression ${ }^{7}$.

In this context, the new study by Skovlund et al. ${ }^{1}$ is remarkable for its breadth of inclusion of contraceptive devices: the investigators include combined oestrogen and progesterone as well as progesterone-only preparations that are administered by means of a host of delivery mechanisms, including oral, transdermal, vaginal, intrauterine and depot forms. The large sample size in this study, which is made possible by Denmark's nationalized information collection systems, is impressive, with a total of 1,061,997 Danish women aged between 15 years and 34 years, excluding only those who had a prior diagnosis or treatment of depression, other psychiatric illness, cancer, venous thrombosis or infertility treatment.

Skovlund and colleagues show that in their population, the risk ratio for first diagnosis of or treatment for depression increases steadily for 6 months after initiation of all forms of hormonal contraception. After 6 months, the risk of first-time diagnosis or treatment decreases slowly, not returning to nonuse levels until 4 years or later. Notably, the risk ratio for use of an antidepressant was found to be highest for adolescents (defined here as age 1519 years), with a 1.8-fold relative risk compared with a control group of adolescent women who were not prescribed hormonal contraceptives. According to these results, for a physician to observe symptoms that would lead to anti-depressant treatment as a result of hormonal contraception in one patient, she or he would need to initiate oral contraceptive treatment in $\sim 225$ adults, or $\sim 95$ adolescents. These numbers are derived from raw data presented in the manuscript, without adjusting for population differences, such as education or calendar year. Interestingly, the investigators also broke down the relative risk posed by each contraceptive by type of hormone and type of delivery system. For example, in adolescents, non-oral methods of both combined and progesterone-only treatment led to around a threefold increased relative risk of either first diagnosis of depression or use of antidepressant medication compared with adolescents who were not prescribed contraception. However, presenting data as relative risk can be misleading, as the incidence of these events is quite low. Put another way, among adolescent girls currently on non-oral contraceptives, one in $\sim 30$ would be started on anti-depressant medications within a year, whereas one in $\sim 150$ would be diagnosed with depression. With population factors adjusted for age, calendar year, educational level, diagnosis of polycystic ovary syndrome and diagnosis of endometriosis, the numbers decrease to one in $\sim 50$ and one in $\sim 250$, respectively. In the general population, $\sim 6 \%$ of female adolescents are diagnosed yearly with depression, and complaint of depressive symptoms is even more common ${ }^{8}$. These data raise the question - are these relative risks a cause for alarm for an individual care provider? 
The authors were not able to control for a few potential interactions. First, the reason for initiating hormonal contraception is a potential contributing or confounding variable, as the reasons underlying this decision might themselves be correlated to depression. This factor could also confound the differential effects seen with the various types of contraceptive use, as illustrated in a 2007 study, which reported that women in the STAR*D trial who were on progestin-only oral contraceptive often had other comorbidities that could have affected their incidence of depression ${ }^{9}$. Another study showed that adolescents with depressive symptoms are more likely to choose an intrauterine device for contraception, which confers a potential bias in cohort studies that could affect the outcome measures ${ }^{10}$. Another missing variable is the potential use of nonhormonal contraception such as a copper intrauterine device, or condom, which could affect outcomes within the control groups. Controlling for these variables would increase the specific utility of this study to individual patients. Finally, the timing of the rise in risk could be an artefact of discontinuation of the hormonal contraceptives: if a woman (or her doctor) was able to correlate the onset of depression with the use of the contraceptive treatment, then she would probably discontinue its use by the 6month time-point. Further study of this population would be interesting to see if the diagnosis of depression persists after removal of the contraceptive treatment.

Whether or not acute use of hormonal contraception (for example, levonorgestrel, also known as Plan B) has any similar effects would be of interest for further study, although it would be important to dissociate this effect from the potential influence of interpersonal interactions related to the need for use of this treatment. Additionally, the investigators did not address the potential for other psychological diagnoses, such as anxiety disorders or eating disorders (which can also be treated with antidepressants, but do not require a diagnosis of depression) to be related to hormonal contraception. This omission might account for some of the discrepancy seen in the raw data, in which far more women were started on antidepressants than were diagnosed with a mood disorder. For psychiatrists, determining whether a subtype of depression exists that is particularly responsive to hormonal changes (analogous to ERBB2 (also known as HER2) in breast cancer) would be interesting, or whether the directionality or timing of the mood response to hormonal contraceptives has a genetic underpinning. If genetic factors exist, they could help to define personalized treatments across the lifespan of these patients.

Importantly, the intriguing results of this study have the potential to affect clinical care, particularly the use of hormonal contraceptives for adolescents. The fact that the risk of diagnosis or treatment of depression is highest for adolescents could reflect the active role of sex steroid hormones in brain development, which probably affect outcomes for emotional processing, leading to the symptoms of a mood disorder. On the basis of this study, the use of progesterone-only or non-oral methods of hormonal contraception for adolescents, given the considerably increased risk in potential depression, would need to be carefully considered and balanced against potential benefits. This study should encourage careful counselling when starting hormonal forms of birth control. 


\section{Acknowledgments}

The authors would like to thank S. Hoeppner, Center for Anxiety and Traumatic Stress Disorders, Department of Psychiatry, Massachusetts General Hospital, Massachusetts, USA, for her assistance with statistical analyses. This work was supported by NIH (NHLBI) T32HL007374 and a BIDMC Psychiatry Junior Faculty fund (R.A.R.), and by NIH (NICHD) R01 HD019938, R01 HD082314 and K12 HD051959 (U.B.K.).

\section{References}

1. Skovlund CW, Mørch LS, Kessing LV, Lidegaard Ø. Association of hormonal contraception with depression. JAMA Psychiatry. 2016; 73:1154-1162. [PubMed: 27680324]

2. Kessler RC, McGonagle KA, Swartz M, Blazer DG, Nelson CB. Sex and depression in the National Comorbidity Survey. I: lifetime prevalence, chronicity and recurrence. J Affect Disord. 1993; 29:85-96. [PubMed: 8300981]

3. Yonkers KA, et al. Symptom-onset dosing of sertraline for the treatment of premenstrual dysphoric disorder: a randomized clinical trial. JAMA Psychiatry. 2015; 72:1037-1044. [PubMed: 26351969]

4. Yonkers KA, et al. Efficacy of a new low-dose oral contraceptive with drospirenone in premenstrual dysphoric disorder. Obstet Gynecol. 2005; 106:492-501. [PubMed: 16135578]

5. Schaffir J, Worly BL, Gur TL. Combined hormonal contraception and its effects on mood: a critical review. Eur J Contracept Reprod Health Care. 2016; 21:347-355. [PubMed: 27636867]

6. Svendal G, et al. The use of hormonal contraceptive agents and mood disorders in women. J Affect Disord. 2012; 140:92-96. [PubMed: 22537684]

7. Andréen L, et al. Sex steroid induced negative mood may be explained by the paradoxical effect

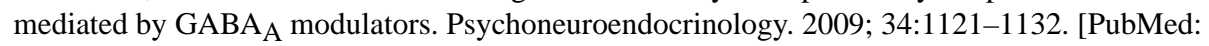
19272715]

8. Jane Costello E, Erkanli A, Angold A. Is there an epidemic of child or adolescent depression? J Child Psychol Psychiatry. 2006; 47:1263-1271. [PubMed: 17176381]

9. Young EA, et al. Influences of hormone-based contraception on depressive symptoms in premenopausal women with major depression. Psychoneuroendocrinology. 2007; 32:843-853. [PubMed: 17629629]

10. Francis J, Presser L, Malbon K, Braun-Courville D, Linares LO. An exploratory analysis of contraceptive method choice and symptoms of depression in adolescent females initiating prescription contraception. Contraception. 2015; 91:336-343. [PubMed: 25553873]

\section{Biographies}

Rachel Ross received her combined MD and PhD in 2010 from Albert Einstein College of Medicine, Bronx, New York, USA. Currently, she is an Instructor in Psychiatry at Harvard Medical School, with a clinic focused on treating people with anxiety disorders, and a research program focused on understanding hypothalamic neurocircuitry involved in metabolism related to body weight, fertility and stress response, and the role that this circuitry plays in psychiatric illness.

Ursula Kaiser received her MD in 1986 from University of Toronto, Canada. Currently, she is Chief of the Division of Endocrinology, Diabetes and Hypertension at Brigham and Women's Hospital and Professor of Medicine at Harvard Medical School in Boston, Massachusetts, USA. She has an active research program focused on the genetic and molecular mechanisms underlying the neuroendocrine control of reproductive development and function. 